\title{
Biometrics Based on Multispectral Skin Texture
}

\author{
Robert K. Rowe \\ Lumidigm, Inc, 801 University Blvd., SE, Suite 302, Albuquerque, NM 87106 \\ Rob. Rowe@Lumidigm. com
}

\begin{abstract}
Multispectral imaging (MSI) of the skin provides information about both the surface and subsurface characteristics of the skin tissue. These multispectral characteristics may be described as textures and used to determine the identity of an individual. In this investigation, a multi-day, multi-person study was conducted to compare the performance of a matcher based on multispectral texture analysis with a conventional minutiae-based fingerprint matcher. Both matchers used MSI data from exactly the same area of the sensor. Performance of the two methods was compared for a range of simulated sensor areas. The performance of the textural matcher was nearly equivalent to that of the minutiae matcher for the larger sensor areas within the limited scope of this study. For small sensor areas, the performance of the texture matcher was significantly better than the minutia matcher operating over the identical small region.
\end{abstract}

Keywords: multispectral skin texture, skin biometrics, multispectral imaging, local consistency.

\section{Introduction}

Fingerprint-based biometric sensors are used across a broad range of applications, from law enforcement and civil identification to commercial access control and even in some consumer devices such as laptops and cell phones. In the latter cases, there is a need to reduce the size of the sensor in order to reduce the area of the device that the sensor occupies and also, generally, to reduce the cost of the sensor. However, the performance of a contact fingerprint sensor degrades as the size decreases [1]. Because of this, some manufacturers [2, 3, 4] produce long and narrow fingerprint sensors that simulate a larger-area sensor by combining a series of narrow images collected while the user "swipes" their finger across the sensor surface. Such a sensor configuration places a burden on the user and limits the applications in which this type of solution is considered.

One way to reduce the sensing area while maintaining a simple, single-touch user interface is to measure a property of the skin that is locally consistent while still being distinct from person to person. In this way, a small-area sensor would be able to perform a biometric match using a skin location never previously enrolled as long as the optical properties of the enrolled and tested skin sites were "similar enough".

In this paper, we examine the feasibility of using multispectral imaging (MSI) for small-area sensors. The matching is done using textural descriptors of the multispectral 
data combined with a classification methodology that seeks to find characteristics of the data that are locally consistent. We test the performance of this matcher against that of a commercial minutiae matcher operating on fingerprint images developed from the same multispectral data used for the texture matcher.

\section{Relationship to Other Work}

A number of papers have been published in the area of using texture matching of fingerprints. For example, Jain [5] proposed a local texture analysis using Gabor filters applied to tessellated regions around the core point. Lee and Wang [6] and Hamamoto [7] also used Gabor filters localized at the core. Coetzee and Botha [8] and Willis and Myers [9] proposed a Fourier analysis fingerprint texture as the basis for biometric determinations. Tico et al. [10] applied wavelet analysis to fingerprint images to distinguish between them. Apart from differences in basis functions and other methodological matters, the key difference between these prior efforts and the one reported in this paper is that, to this investigator's knowledge, all prior investigations are based on conventional fingerprint images. As such, the observed textural pattern is extracted from a single image, which limits the information content and can be adversely affected by artifacts due to effects such as dry skin, poor contact between the skin and sensor, and other operationally important matters. In contrast, the present investigation is based on multiple images taken with a robust imaging methodology which contains information about both the surface and subsurface characteristics of the skin while minimizing sampling artifacts. In fact, the one data plane in the MSI stack most similar to conventional optical fingerprinting techniques was explicitly removed from the texture analysis in order to avoid the presence of spurious effects, as described later in this paper.

The topic of how to perform biometric matching using small-area fingerprint sensors is an active area of investigation. One common approach is to build up a large enrollment image by piecing together a series of small fingerprint images taken over multiple placements of the finger in a process known as mosaicing [1, 11]. Alternatively, some authors have combined the minutiae information together instead of the image themselves $[12,13,14,15]$. In contrast to either of these approaches, the work described in this paper is associated with finding those characteristics of the multispectral skin texture that are locally consistent in order that an enrollment measurement may be made at one skin site and successfully verified at a different but nearby skin site.

One manufacturer of a facial recognition system [16] says they use "surface texture analysis" to augment and improve the performance of their commercial system. The major difference between such a surface-based method and the methods and systems described in this paper is that the MSI sensor has been developed to obtain a significant portion of information from features below the surface of the skin. Moreover, the plurality of wavelengths, illumination angles, and optical polarization conditions used in this investigation yields additional information beyond that available through simple surface reflectance measurements. 


\section{Multispectral Skin Sensing}

\subsection{Hardware and Raw MSI Data}

In order to capture information-rich data about the surface and subsurface features of the skin of the finger, the MSI sensor collects multiple images of the finger under a variety of optical conditions. The raw images are captured using different wavelengths of illumination light, different polarization conditions, and different illumination orientations. In this manner, each of the raw images contains somewhat different and complementary information about the finger. The different wavelengths penetrate the skin to different depths and are absorbed and scattered differently by various chemical components and structures in the skin. The different polarization conditions change the degree of contribution of surface and subsurface features to the raw image. Finally, different illumination orientations change the location and degree to which surface features are accentuated.

Fig. 1 shows a simplified schematic of the major optical components of an MSI fingerprint sensor. Illumination for each of the multiple raw images is generated by one of the light emitting diodes (LEDs). The figure illustrates the case of polarized, direct illumination being used to collect a raw image. The light from the LED passes through a linear polarizer before illuminating the finger as it rests on the sensor platen. Light interacts with the finger and a portion of the light is directed toward the imager through the imaging polarizer. The imaging polarizer is oriented with its optical axis to be orthogonal to the axis of the illumination polarizer, such that light with the same polarization as the illumination light is substantially attenuated by the polarizer. This severely reduces the influence of light reflected from the surface of the skin and emphasizes light that has undergone multiple optical scattering events after penetrating the skin.

The second direct-illumination LED shown in Fig. 1 does not have a polarizer placed in the illumination path. When this LED is illuminated, the illumination light is randomly polarized. In this case the surface-reflected light and the deeply penetrating light are both able to pass through the imaging polarizer in equal proportions. As such, the image produced from this non-polarized LED contains a much stronger influence from surface features of the finger.

Importantly, all of these direct-illumination sources (both polarized and nonpolarized) as well as the imaging system are arranged to avoid any critical-angle phenomena at the platen-air interfaces. In this way, each illuminator is certain to illuminate the finger and the imager is certain to image the finger regardless of whether the skin is dry, dirty or even in contact with the sensor. This aspect of the MSI imager is distinctly different from most other conventional fingerprint imaging technologies and is a key aspect of the robustness of the MSI methodology.

In addition to the direct illumination illustrated in Fig. 1, the MSI sensor also integrates a form of total internal reflectance (TIR) imaging. In this illumination mode, one or more LEDs illuminate the side of the platen. A portion of the illumination light propagates through the platen by making multiple TIR reflections at the platen-air interfaces. At points where the TIR is broken by contact with the skin, light enters the skin and is diffusely reflected. A portion of this diffusely reflected light is directed toward the imaging system and passes through the imaging polarizer 


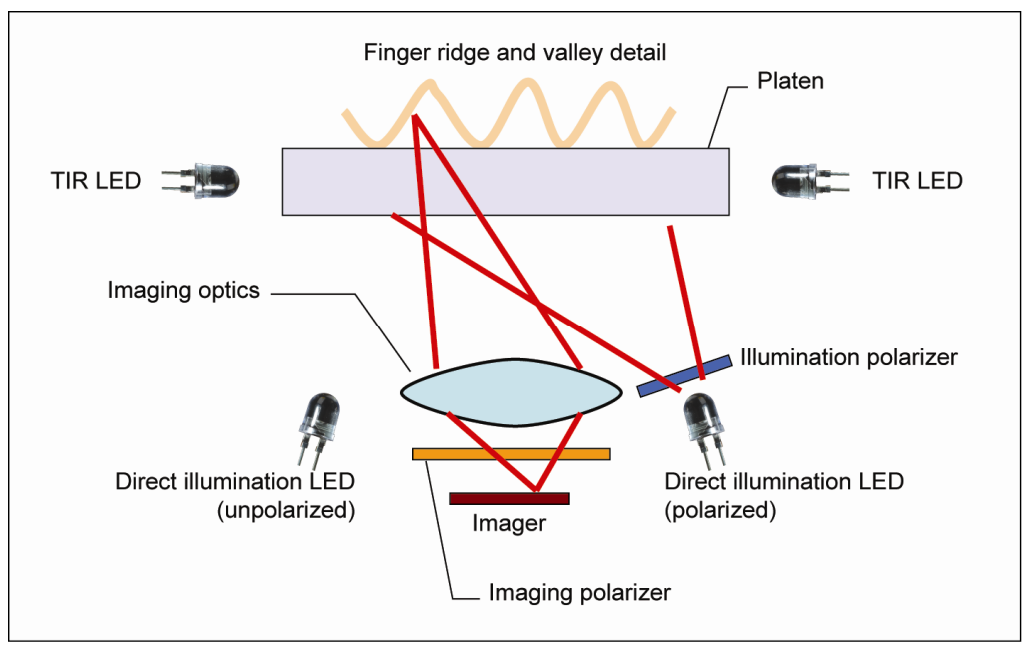

Fig. 1. Optical configuration of an MSI sensor. The red lines illustrate the direct illumination of a finger by a polarized LED.

(since this light is randomly polarized), forming an image for this illumination state. Unlike all of the direct illumination states, the quality of the resulting raw TIR image is critically dependent on having skin of sufficient moisture content and cleanliness making good optical contact with the platen, just as is the case with conventional TIR sensors.

In practice, MSI sensors typically contain multiple direct-illumination LEDs of different wavelengths. For example, the Lumidigm J110 MSI sensor used to collect the data used in this investigation has four direct-illumination wavelength bands (430, 530, and $630 \mathrm{~nm}$ as well as a white light) in both polarized and unpolarized configurations. When a finger is placed on the sensor platen, eight direct-illumination images are captured along with a single TIR image. The raw images are captured on a $640 \times 480$ image array with a pixel resolution of 525 ppi. All nine images are captured in approximately $500 \mathrm{mSec}$.

An example of the nine images captured during a single finger placement is illustrated in Fig 2. The upper row shows the raw images for unpolarized illumination wavelengths of 430, 530, and $630 \mathrm{~nm}$, as well as white light. The lower row shows the corresponding images for the cross-polarized case as well as the TIR image. The grayscale for each of the raw images has been expanded to emphasize the features.

\subsection{Composite Fingerprint Generation and Matching}

It can be seen from Fig. 2 that there are a number of features present in the raw data including the textural characteristics of the subsurface skin, which appears as mottling that is particularly pronounced under blue $(430 \mathrm{~nm})$ and green $(530 \mathrm{~nm})$ illumination wavelengths. As well, the relative intensities of the raw images under each of the illumination conditions is very indicative of the spectral characteristics (i.e. color) of the finger or other sample (note that the relative intensities have been obscured in Fig. 2 to better show the comparative details of the raw images). 


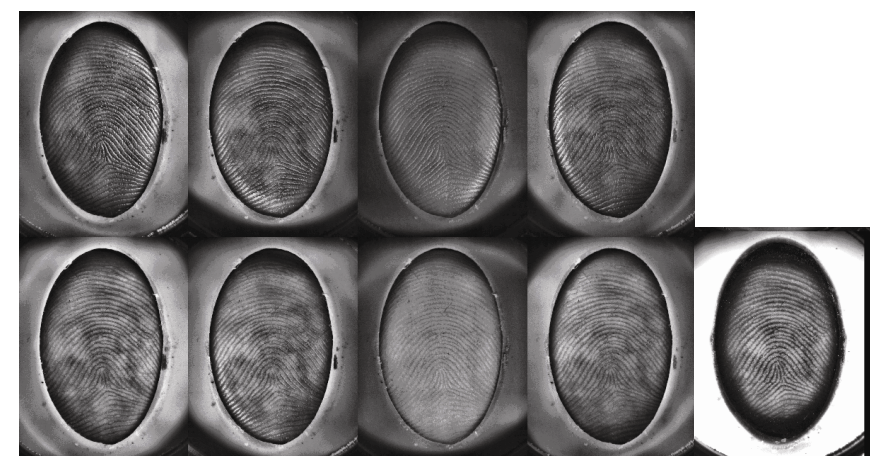

Fig. 2. Raw MSI images. The upper row of images corresponded to non-polarized illumination of various wavelengths (from left to right: 430, 530, 630nm and white light). Directly below each of these images is the corresponding cross-polarized illumination case. The image at the extreme right of the lower row is the TIR image.

The set of raw images shown in Fig. 2 can be combined together to produce a single representation of the fingerprint pattern. This fingerprint generation relies on a wavelet-based method of image fusion to extract, combine and enhance those features that are characteristic of a fingerprint. The wavelet decomposition method that is used is based on the dual-tree complex wavelet transform (DTCWT) [17]. Image fusion occurs by selecting and compiling the coefficients with the maximum absolute magnitude in the image at each position and decomposition level [18]. An inverse wavelet transform is then performed on the resulting collection of coefficients, yielding a single, composite image. An example of the result of applying the compositing algorithm to two placements of the same finger is given in Fig. 3 .

The composite fingerprint images can then be used with conventional fingerprint matching software. For this investigation, a commercial minutiae-based feature extraction and matching routine (NEC, NECSAM FE4, ver. 1.0.2.0, PPC2003) was applied to the composite fingerprints as a control to compare to texture matching results.
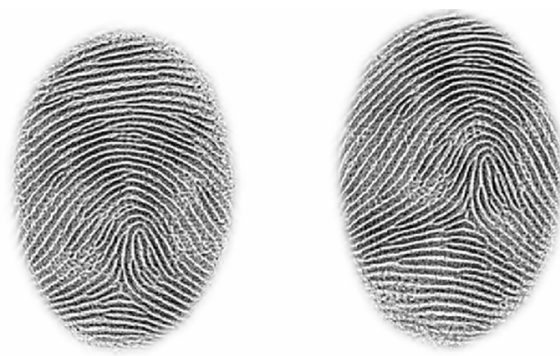

Fig. 3. Composite fingerprint images extracted from 2 different sets of multispectral data like those shown in Fig. 2 


\subsection{Multispectral Texture Analysis}

The DTCWT process that was used to generate composite fingerprint images was also used to provide the spectral-textural features of the multispectral data. The coefficients from the third level of the DTCWT decomposition of the multispectral image stack were used as features for the texture analysis in this investigation. Since the strength and quality of the raw TIR image plane is highly variable (dependent on skin moisture, good contact, etc.), this plane was omitted from the multispectral texture analysis but not from the corresponding composite fingerprint.

Anderson et al. [19] have defined a set of image features based on a DTCWT decomposition that they refer to as inter-level products. These features represent the conjugate products of the DTCWT coefficients in two adjacent decomposition levels. They have also defined an inter-coefficient product (elsewhere in their publications it is referred to as "same-level product") that represents the conjugate product of adjacent coefficients in the same decomposition level. These conjugate products have been shown to represent the fundamental features of the image while being insensitive to translation and some amount of rotation.

In a similar way, we define an inter-image product, $P$, as the conjugate product of coefficients at some direction, $d$, decomposition level, $k$, generated by any 2 of the raw multispectral images, $i$ and $j$, in a multispectral image stack at location $x, y$ :

$$
P_{i, j}(x, y, d, k)=C_{i}(x, y, k) C_{j}^{*}(x, y, k),
$$

where $C_{i}(x, y, k)$ is the complex coefficient for image $\mathrm{i}$ at decomposition level $\mathrm{k}$ and location $\mathrm{x}, \mathrm{y}$ while $C^{*}{ }_{j}(x, y, k)$ is the conjugate of the corresponding complex value for image $\mathrm{j}$.

For purposes of this investigation, we compiled all real and imaginary components of all the conjugate products generated from each unique image pair as a feature vector. For 8 raw image planes, this results in a 384 element vector (28 conjugate products/direction, 6 directions, 2 scalar values (real and imaginary) per product for $i \neq j$, plus 8 conjugate products/direction, 6 directions, 1 scalar value (real only) for $i=j$ ). In addition, the isotropic magnitudes of the coefficients were added to the feature vector, where the isotropic magnitude is simply the sum of the absolute magnitudes over the 6 directional coefficients. Finally, the mean DC values of each of the raw images over the region of analysis were added to the feature vector. Concatenating all of these values resulted in a 400-element feature vector at each element location.

\section{Experimental Data and Analysis}

Multispectral data were collected on 21 volunteers over a period of approximately 2 weeks. The participants were office workers whose ages ranged from the mid-20s to the mid-60s. They were of mixed gender and race. Each participant made two visits separated by at least one day. During a visit, each of the 4 fingers on the right hand was measured 3 times, yielding 12 multispectral data sets collected per person per visit. This resulted in a total dataset comprised of 504 multispectral image stacks taken over 84 unique fingers. 
For purposes of this investigation, the data collected during a participant's first visit was treated as enrollment data and data from the second visit was used for testing. A summary of the spans of time between enrollment and verification for the 21 study participants is: 1 day (16 people), 3 days ( 2 people), 4 days ( 1 person), 5 days ( 1 person) and 11 days ( 1 person).

Since the dataset used for this analysis was of a relatively modest size, the biometric task that was tested was chosen to be personalization, which requires far less data than verification (or any other "open" formulation) to obtain stable results. In this testing scenario, there are $\mathrm{N}$ enrolled people and each test sample is assumed to be one of the enrollees. No attempt is made to determine or discriminate against nonenrolled test samples. The estimate for identity is simply the enrollment sample that is the closest match to the test sample.

Multiple, random trials were conducted to determine the performance of both the multispectral texture matcher as well as the fingerprint matcher used as a control for each tested condition. Each trial was based on enrollment by 6 randomly selected fingers from 6 randomly selected people. These same data were also used to build a classification model as described below. The data from the same fingers taken during the second visit for each of the 6 enrolled fingers were used for performance testing. In order to augment the calibration model, the data from one randomly selected finger from each of the other 15 people in the dataset who were not enrolled for a particular trial were included as part of the calibration dataset.

Multispectral texture analysis was performed on an element-by-element basis over a particular analysis region. Each analysis region comprised a contiguous area of the imager with pixel dimensions of $64 \times 48$. The number of analysis regions used for each of the 4 cases that were studied varied from $5 \times 5$ (320 x 240 pixels, 0.61 " $\mathrm{x}$ $0.46 ")$ to 1 x 1 (64 x 48 pixels, $0.12 "$ x 0.09 "). In each of the cases studied, the comparison was made to a fingerprint that was generated from the multispectral data and masked to cover exactly the same area as used for the multispectral match. The four cases are illustrated and further described in Fig. 4. For all cases, the analysis regions were centered on the active image area.

The calibration data for a given analysis region was used as an input into a Fisher linear discriminant analysis (FLDA) algorithm to develop a classification model. Prior to processing the calibration data, the data were normalized such that the standard deviation of each of the elements of the feature vector was 1.0 over the calibration set. The same normalization factor was also applied to the test data.

The test samples were then matched to the enrollment samples by projecting the difference in feature vectors onto the FLDA factors and accumulating an RMS summary of the resulting projection. The differences were accumulated for all elements within an analysis region and for all analysis regions used in a particular case. The estimate of the identity of the test sample was then selected as the enrollment that had the smallest accumulated match value associated with it.

The comparison to fingerprint minutiae matching was done by masking the MSIderived fingerprint to the same area as used for the corresponding MSI texture analysis. The minutiae matching was then performed between the test image and the $6 * 3=18$ enrolled images. The ID of the enrolled image with the largest match value was recorded for that particular test image. 

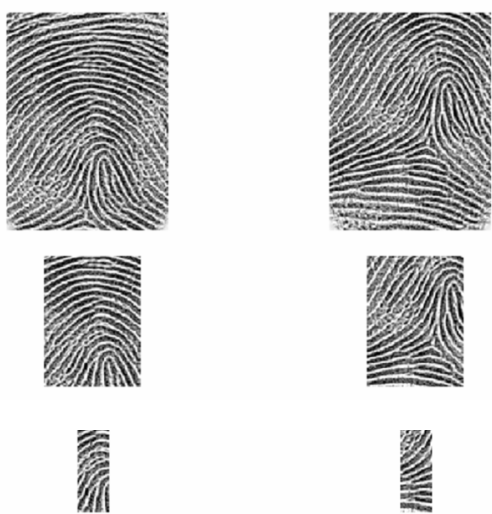

8

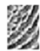

Fig. 4. Illustration of sensor sizes used in this study using the same images as in Fig 3. The largest sensor size is at the top and is 320 x 240 ( 0.61 " x 0.46 ") followed by 192 x 144 ( 0.37 " $\mathrm{x}$ $0.27 "), 192 \times 48$ (0.37" x 0.09") and 64 x 48 (0.12" x 0.09 ") on the bottom.

\section{Results}

The performance of each test configuration was estimated by aggregating the results of 20 random trials conducted for each of the test conditions. The summary of these results is given in Fig. 5.

A comparison of the results of this study indicates that the performance of the multispectral texture matcher is approximately the same as the fingerprint matcher for
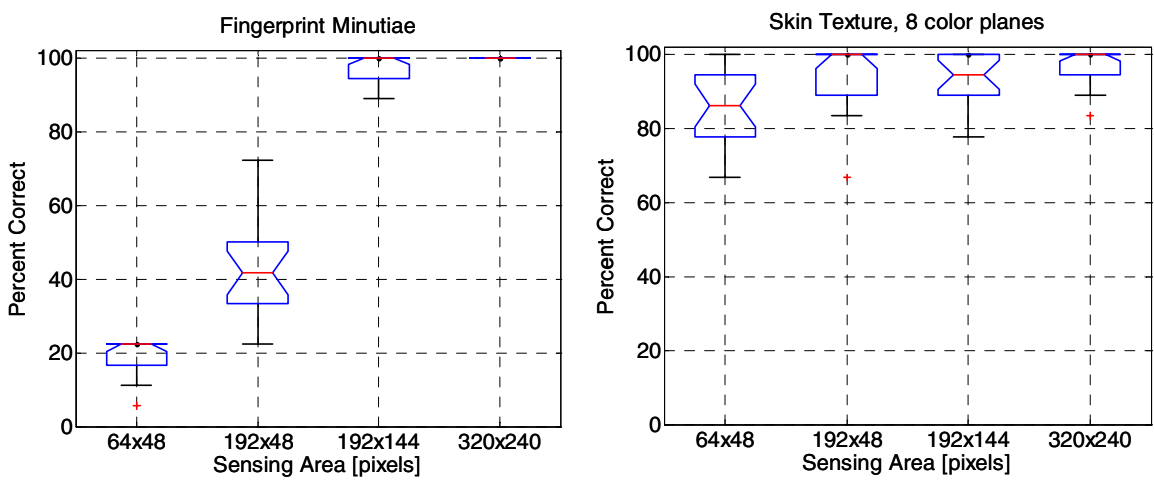

Fig. 5. Results for fingerprint matching (left) and multispectral skin texture matching (right) for 4 different sensor sizes. The boxes indicate the inner two quartiles of the 20 random trials and the red line is the median of the 20 trials. The notch indicates the uncertainty of the median at a $5 \%$ significance level. 
the two largest sensor areas that were tested. Further work with larger datasets will be required to quantify the performance differences between a minutiae matcher and a multispectral texture matcher over the same, relatively large area of the finger.

However, the results from this modestly sized study do indicate that as the sensor size decreases, the performance of the texture-based matcher is maintained better than that of the minutiae-based matcher, which degrades sharply for the smallest sensor areas tested. This difference in performance is possibly due to the property of local consistency of the multispectral texture: skin proximal to the point of enrollment has approximately the same properties as the enrollment site itself. Therefore, placementto-placement variation (which becomes more severe as the sensor size decreases) affects the texture matcher far less than the minutiae matcher.

\section{References}

1. Maltoni, D., Maio, D., Jain, A.K., Prabhakar, S.: Handbook of Fingerprint Recognition. Springer, Heidelberg (2003)

2. http://www.authentec.com

3. http://www.upek.com

4. http://www.atmel.com/products/biometrics/

5. Jain, A.K., Prabhakar, S., Hong, L., Pankanti, S.: Filterbank-based fingerprint matching. IEEE Transactions on Image Processing 9, 846-859 (2000)

6. Lee, C.J., Wang, S.D.: Fingerprint feature extraction using Gabor filters. Electronic Letters 35(4), 288-290 (1999)

7. Hamamoto, Y.: A Gabor filter-based method for fingerprint identification. In: Jain, L.C., Halici, U., Hayashi, I., Lee, S.B. (eds.) Intelligent Biometric Techniques in Fingerprint and Face Recognition, CRC Press, Boca Raton, FL (1999)

8. Coetzee, L., Botha, E.C.: Fingerprint recognition with a neural-net classifier. In: Proc. South African Workshop on Pattern Recognition, 1st edn. vol. 1, pp. 33-40 (1990)

9. Willis, A.J., Myers, L.: A cost-effective fingerprint recognition system for use with lowquality prints and damaged fingertips. Pattern Recognition 34(2), 255-270 (2001)

10. Tico, M., Kuosmanen, P., Saarinen, J.: Wavelet domain features for fingerprint recognition. Electronics Letters 37(1), 21-22 (2001)

11. Brown, L.G.: Image registration techniques. ACM Computing Surveys 24(4), 326-376 (1992)

12. Yau, W.Y., Toh, K.A., Jiang, X., Chen, T.P., Lu, J.: On fingerprint template synthesis. In: Proc. Int. Conf. on Control Automation Robotics and Vision, 6th edn. (2000)

13. Toh, K.A., Yau, W.Y., Jiang, X., Chen, T.P., Lu, J., Lim, E.: Minutiae data synthesis for fingerprint identification applications. In: Proc. Int. Conf. on Image Processing, vol. 3, pp. 262-265 (2001)

14. Jain, A.K., Ross, A.: Fingerprint mosaicking. In: Proc. Int. Conf. on Acoustic Speech and Signal Processing, vol. 4, pp. 4064-4067 (2002)

15. Ramoser, H., Wachmann, B., Bischof, H.: Efficent alignment of fingerprint images. In: Proc. Int. Conf. on Pattern Recognition (16th), vol. 3, pp. 748-751 (2002)

16. http://www.visionics.com/trends/skin.html 
17. Kingsbury, N.: Complex wavelets for shift invariant analysis and filtering of signals. Journal of Appl. and Comput. Harmonic Analysis 10, 234-253 (2001)

18. Hill, P., Canagarajah, N., Bull, D.: Image fusion using complex wavelets. In: Proc. 13th British Machine Vision Conference, Cardiff, UK (2002)

19. Anderson, R., Kingsbury, N., Fauqueur, J.: Robust rotation-invariant object recognition using edge-profile clusters. submitted to European Conference on Computer Vision (May 2006) 